\title{
Magnetic Resonance Neurography
}

Authors: Avneesh Chhabra and Gustav Andreisak

Publisher: Jaypee Brothers Medical Publishers Pvt Ltd.

Edition: $1^{\text {st }}$

Pages: 218; Price: INR:1995 and \$ 125

Magnetic

RiEONANGE
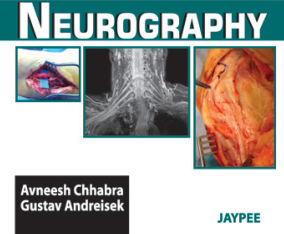

Magnetic Resonance Neurography by Avneesh Chhabra and Gustav Andreisak is a comprehensive and eminently readable textbook that would appeal to practicing radiologists with an interest in neuroimaging and musculoskeletal imaging, as well as residents and technologists.

The textbook is broadly divided into nine sections. The first three chapters address the peripheral nerve anatomy, pathophysiology, techniques of magnetic resonance neurography (MRN), and principles of general interpretation. Chapters 4 and 5 are dedicated to the upper and lower extremities; Chapter 6 addresses neurogenic tumors; and Chapters 7 and 8 address the brachial and lumbosacral plexuses, respectively. The textbook ends with a section devoted to MRI injections focused on perineural and muscle medication injections.

The salient feature of the textbook is the section on MRI techniques that describes the pulse sequences and imaging planes used to obtain high-resolution and clinically relevant images, including practical considerations such as the appropriate use of intravenous contrast.

The reader is then taken through interpretation of the generated MRN images, including differential diagnosis, and also familiarized with the currently practiced management algorithms. Nerve anatomy, including normal variations and muscle innervation patterns, are explained.

The final chapter focuses on diagnostic and therapeutic selective peripheral nerve injections, involving imaging guided delivery of local anesthetics and steroids.

The language is lucid, the diagrams are easy to comprehend and clearly labeled. The text is liberally accompanied by high-resolution images.

The textbook design is compact, user friendly, and visually appealing.

Darshana Sanghvi

Kokilaben Dhirubhai Ambani Hospital, Rao Saheb Achutrao Patwardhan Marg, Four Bunglows, Andheri (W), Mumbai - 400 053, Maharashtra, India. E-mail: sanghvidarshana@gmail.com

\begin{tabular}{|l|l|}
\hline \multicolumn{2}{|c|}{ Access this article online } \\
\hline Quick Response Code: & \multirow{2}{*}{ Website: } \\
\hline & www.ijri.org \\
\hline
\end{tabular}

